\title{
Hepatic stimulator substance inhibits calcium overflow through the mitochondria-associated membrane compartment during nonalcoholic steatohepatitis
}

\author{
Fan Xiao ${ }^{1,2}$, Jing Zhang ${ }^{1}$, Can Zhang ${ }^{1}$ and Wei $A n^{1}$
}

Nonalcoholic fatty liver disease is considered a disorder of the endoplasmic reticulum (ER) and mitochondria. Recent studies have shown that the ER and mitochondrial membranes overlap by $15-20 \%$, a region referred to as the 'mitochondria-associated ER membrane' (MAM). Some proteins, including sarco/ER calcium ATPase (SERCA), are located in the MAM and have an important role in $\mathrm{Ca}^{2+}$ signaling and homeostasis between the ER and the mitochondria. Our previous study showed that hepatic stimulator substance (HSS) inhibits the ER stress induced by reactive oxygen species, thus reducing mitochondrial damage. However, the mechanism underlying the protective effect of HSS on the ER and ERmitochondrial interaction remains unclear. In this study, we confirmed that the exogenous expression of HSS protected the liver from steatosis in mice with nonalcoholic steatohepatitis. More importantly, the protection provided by HSS allowed SERCA in the MAM compartment to function well, preventing the extensive influx of cytosolic free $\mathrm{Ca}^{2+}$ to the mitochondria, thus preserving the mitochondrial functions from calcium overload and relieving palmitic-acid-induced hepatocyte steatosis. Our results suggest that the protective effect of HSS on SERCA expression is associated with the maintenance of calcium homeostasis within the MAM, thus ameliorating the disordered $\mathrm{Ca}^{2+}$ communication between the ER and mitochondria.

Laboratory Investigation (2017) 97, 289-301; doi:10.1038/labinvest.2016.139; published online 19 December 2016

Nonalcoholic fatty liver disease (NAFLD) is a spectrum of diseases ranging from simple steatosis to nonalcoholic steatohepatitis (NASH), which involves more severe hepatic lesions and marked inflammation, than fibrosis, and also endstage liver disease, such as cirrhosis. ${ }^{1}$ NAFLD is now recognized as one of the most common causes of liver disease in the world. ${ }^{2}$ The endoplasmic reticulum (ER), one of the largest cellular organelles, is responsible for the proper assembly and posttranslational modification of proteins destined for intracellular organelles and the cell surface. The ER is also the main storage site for cytosolic calcium. The ER $\mathrm{Ca}^{2+}$ levels are controlled by the balance between calcium release and calcium uptake, which has an important role in the regulation of ER homeostasis. The disruption of ER homeostasis, often called 'ER stress', has been observed in the livers of humans with NAFLD and also in the experimental rodents. ${ }^{3}$ Therefore, it is strongly believed that ER stress and the subsequent response to it, called the 'unfolded protein response', have important roles in the pathogenesis of NAFLD and may be critical factors in the progression of NAFLD to NASH. ${ }^{4}$ Therefore, the mechanisms that disrupt the $\mathrm{Ca}^{2+}$ equilibrium of the ER and disturb ER-mediated $\mathrm{Ca}^{2+}$ signaling, which may exacerbate the progression of NAFLD, have become topics of intense investigation. It also appears that the ER and mitochondria interact both physically and functionally via a special structure known as the 'mitochondria-associated membrane' (MAM), ${ }^{5}$ which allows the ERmediated transfer of calcium to the mitochondria, altering the mitochondrial functions and oxidative stress in NAFLD. So far, a large number of proteins have been have identified in the MAM compartment, including the mitochondrial voltagedependent anion channel and sarco/ER calcium ATPase (SERCA), which regulate the $\mathrm{Ca}^{2+}$ flow between the ER and mitochondria. ${ }^{6}$ During ER stress, the transient overflow of calcium transmitted from the ER to the adjacent mitochondrial network via the MAM pathway is particularly harmful to

${ }^{1}$ Department of Cell Biology and Municipal Laboratory for Liver Protection and Regeneration Regulation, Capital Medical University, Beijing, China

Correspondence: W An, PhD, Department of Cell Biology and Municipal Laboratory for Liver Protection and Regeneration Regulation, Capital Medical University, 10 You An Men Wai Xi Tou Tiao, Fengtai District, Beijing 100069, China.

E-mail: anwei@ccmu.edu.cn

${ }^{2}$ Current address: Division of Infectious Diseases, Beijing Ditan Hospital, Capital Medical University, Beijing 100015, China.

Received 24 June 2016; revised 26 October 2016; accepted 17 November 2016 
the mitochondria because this $\mathrm{Ca}^{2+}$ wave, or eventual $\mathrm{Ca}^{2+}$ overload if sustained, can impair oxidative phosphorylation and ATP production, and even trigger mitochondrial apoptosis. ${ }^{7}$ Knowledge of the MAM- $\mathrm{Ca}^{2+}$ signaling pathway and its effect on the mitochondria is essential for understanding the development of NAFLD.

Hepatic stimulator substance (HSS) was first described in the liver cytosol of a weanling rat by LaBrecque and Pesch ${ }^{8}$ in 1975. HSS stimulates hepatic DNA synthesis and therefore promotes liver regeneration after partial hepatectomy. ${ }^{9}$ The effect of HSS on hepatocytes is believed to be a liver-specific and non-species-specific phenomenon. ${ }^{10}$ HSS was subsequently shown to protect hepatocytes from toxic agents, such as $\mathrm{CCl}_{4},{ }^{11}$ D-galactosamine, ${ }^{12}$ and $\mathrm{H}_{2} \mathrm{O}_{2} \cdot{ }^{13}$ HSS also confers resistance to hepatic ischemia-reperfusion injury by protecting mitochondrial functions, ${ }^{14}$ and the protective effects of HSS on liver functions may be related to its improvement of mitochondrial functions. ${ }^{15,16}$ Despite the HSS nomenclature, an earlier study showed that HSS has little effect on stimulating the growth of primary cultured hepatocytes, but can augment the proliferative effects of other mitogens, such as epidermal growth factor. Based on this property, HSS is also known as augmenter of liver regeneration (ALR). ${ }^{17}$ The conserved $\mathrm{C}_{62}-\mathrm{X}-\mathrm{X}-\mathrm{C}_{65}(\mathrm{CxxC})$ motif within the C-terminal region of the ALR protein molecule is required for its role in mitochondrial protection. ${ }^{18}$ In a recent study, we also demonstrated that HSS inhibits ER stress and protects SERCA activity against injury by reactive oxygen species (ROS), thus preventing cell damage. ${ }^{19}$ The molecular features of ALR have been extensively investigated and the crystal structure of the ALR protein indicates that it is a sulfhydryl oxidase with a FAD-binding site and a conserved proximal $\mathrm{CxxC}$ motif. ${ }^{20}$ Because mammalian $A L R$ is highly homologous to the yeast Saccharomyces cerevisiae ERV1 gene, originally called 'essential for respiration and viability', ${ }^{21}$ it is also known as 'growth factor, ERV1 homolog of S. cerevisiae' (GFER). In mammals, ALR messenger RNA is expressed in multiple organs, ${ }^{22}$ and the protein is mainly located in the intermembrane space of the mitochondria, ${ }^{23}$ where it seems to be associated with the electron transfer in the respiratory chain. ${ }^{24}$ Its association with the mitochondria and the regulation of ATP synthesis make ALR critically important in the survival of hepatocytes and the inhibition of its expression causes cell death. ${ }^{25}$

We have previously demonstrated that the inhibition of ER stress by HSS may protect the mitochondrial. However, the mechanisms involved remain unclear. We speculate that the ER protection provided by HSS could be associated with the blockage of $\mathrm{Ca}^{2+}$ overflow from the ER to the mitochondria, stimulating the mitochondrial metabolism. If this is the case, the regulation of this activity, particularly during the progression of NAFLD, is an important area of research.

Here, we demonstrate that ER stress occurs during the progression of NAFLD, when SERCA activity at the MAM is severely impaired and mitochondria are damaged and become dysfunctional. Parallel with this effect on SERCA, mitochondrial $\mathrm{Ca}^{2+}$ overload occurs, and the mitochondrial functions are further impaired by lipotoxicity. However, the exogenous expression of HSS restored SERCA activity and prevented mitochondrial $\mathrm{Ca}^{2+}$ overload, possibly improving intracellular $\mathrm{Ca}^{2+}$ homeostasis and alleviating hepatic steatosis.

\section{MATERIALS AND METHODS Reagents}

Dulbecco's modified Eagle's medium and fetal bovine serum were purchased from Gibco (Paisley, UK) and Hyclone (Victoria, Australia), respectively. Antibodies directed against glucose-regulated protein 78 (GRP78, $78 \mathrm{kDa}$, Cat. No. 3183), B-cell leukemia/lymphoma-2 (BCL-2, $26 \mathrm{kDa}$, Cat. No. 15071) were purchased from Cell Signaling Technology (Danvers, MA, USA). Acyl-CoA synthetase 4 (FACL-4, $79 \mathrm{kDa}$, Cat. No. ab155282) was purchased from Cell Signaling Technology (Cambridge, UK). Antibodies directed against HSS fragment (23 kDa, Cat. No. sc-365886), SERCA2 (100 kDa, Cat. No. sc-8094), C/EBP homologous protein (CHOP, $27 \mathrm{kDa}$, Cat. No. sc-7351), $\beta$-actin (42 kDa, Cat. No. sc-47778), protein disulfide isomerase (PDI, $55 \mathrm{kDa}$, Cat. No. sc-74551), cytochrome $c$ (Cytc, $14 \mathrm{kDa}$, Cat. No. sc-13156), and BAX (20 kDa, Cat. No. sc-23959) were purchased from Santa Cruz Biotechnology (Dallas, TX, USA). A triglyceride (TG) quantification kit was purchased from Applygen (Beijing, China). A mitochondrion isolation kit was purchased from Applygen. A mitochondrial membrane potential (MMP) detection kit, mitochondrial permeability transition (MPT) and complex V (ATPase) activity detection kit were purchased from Genmed Company (Shanghai, China). A CellTiter-Glo Luminescent Cell Viability Assay kit was purchased from Promega (Madison, WI, USA). An endoplasmic reticulum isolation kit was purchased from SigmaAldrich (St. Louis, MO, USA). A Ca ${ }^{2+}$-ATPase assay kit was purchased from Jiancheng (Nanjing, China). 1, 2-Bis (2aminophenoxy) ethane- $N, N, N^{\prime}, N^{\prime}$-tetraacetic acid acetoxymethyl ester (BAPTA-AM) was purchased from SigmaAldrich. Methionine/choline-deficient (MCD) diet was purchased from Huafukang Company (Beijing, China).

\section{Preparation of HSS-Bearing Adenovirus}

Human HSS cDNA tagged with FLAG was used to construct a replication-deficient adenoviral vector, pAdxsi (Chinese National Human Genome Centre, Beijing, China), as described previously. ${ }^{14}$ Successive steps in its construction were routinely checked with viral titration and the final product was designated 'Ad-HSS'. The adenoviral vector, pAdxsi, lacking the HSS gene, was designated 'Ad-Null'.

\section{Animal Studies}

Male C57BL/6 mice (6-7 weeks old) were supplied by the Experimental Animal Centre, Capital Medical University, Beijing, China. Totally, 48 animals were applied in the study and they were divided into 4 groups. Each group contained 12 
animals. The mice in the group one were the control and fed with a regular diet, and mice in other three groups (designated as the HSS-transfected, vector-transfected and control-transfected group) were fed with a methionine and choline-deficient (MCD) diet. The mice were transfected with $500 \mu$ l of either the Ad-HSS or Ad-Null virus $\left(1 \times 10^{9}\right.$ plaqueforming units) via the tail vein. The control-transfection was subjected to normal saline injection. The mice in every group were killed either at the end of the first week $(n=6)$ or the second week $(n=6)$. All the animals received humane care in compliance with the Guidelines of the Committee for Institutional Animal Care and Use of Capital Medical University.

\section{Biochemical and Histological Analyses}

The serum concentrations of alanine aminotransferase (ALT), aspartate aminotransferase (AST), TG, and cholesterol were measured. The liver tissues were fixed in $4 \%$ paraformaldehyde, embedded in paraffin, and stained with hematoxylin and eosin. A portion of each liver sample was used to prepare frozen tissue sections, which were stained with oil red O. The rest of the liver tissue was stored at $-80^{\circ} \mathrm{C}$ for further analysis.

\section{Cell Culture}

Hepatocellular carcinoma cell line, BEL-7402, was obtained from the Cell Bank of the Chinese Academy of Sciences (Shanghai, China). The HepG2 cell was obtained from the American Type Culture Collection (Manassas, VA, USA). The BEL-7402 or HepG2 cells were transfected with an HSScontaining plasmid (designated 'HSS-Tr/7402' or 'HSS-Tr/ HepG2') and the control cells were transfected with empty plasmid (designated 'vector-Tr/7402' or 'vector- $\mathrm{Tr} / \mathrm{HepG2}$ '), which are maintained in our laboratory. Untransfected BEL-7402 or HepG2 cells were considered wild-type cells. The cells were cultured in Dulbecco's modified Eagle's medium supplemented with $10 \%$ fetal bovine serum, and the stably HSS-transfected cells were screened with gentamicin (G418, $300 \mu \mathrm{g} / \mathrm{ml}$ for 2 months). The resistant clones were isolated, and the relevant cells were cultured at $37^{\circ} \mathrm{C}$ in an incubator under a humidified atmosphere of $5 \% \mathrm{CO}_{2}$.

\section{Isolation of Mitochondria}

Approximately $3 \times 10^{7}$ cells were homogenized in mitochondrial isolation buffer (10 mM Tris ( $\mathrm{pH} 7.4), 250 \mathrm{mM}$ sucrose, $0.15 \mathrm{mM} \mathrm{MgCl}_{2}$ ). The homogenate was centrifuged at $800 \times g$ at $4{ }^{\circ} \mathrm{C}$ for $5 \mathrm{~min}$, and the supernatant was centrifuged at $10000 \mathrm{~g}$ for $100 \mathrm{~min}$ to obtain a mitochondria-enriched pellet. The pellets were then centrifuged at $12000 \mathrm{~g}$ for $10 \mathrm{~min}$, pooled, washed, and resuspended in isolation buffer for activity measurements.

\section{Assay of Complex V (ATPase) Activity}

This assay linked ATPase activity to nicotinamide adenine dinucleotide (NADH) oxidation via the conversion of phosphoenolpyruvate to pyruvate by pyruvate kinase, and then from pyruvate to lactate by lactate dehydrogenase. The reaction buffer consisted of $250 \mathrm{mM}$ sucrose, $5 \mathrm{mM} \mathrm{MgCl}$, $2 \mathrm{mM} \mathrm{KCN}, 50 \mathrm{mM} \mathrm{KCl}$, and $20 \mathrm{mM}$ Tris- $\mathrm{HCl}$ (pH 7.5). Before the assay, $0.25 \mathrm{mM} \mathrm{NADH}, 1 \mathrm{mM}$ phosphoenol pyruvate, $2.5 \mathrm{U} / \mathrm{ml}$ lactate dehydrogenase, and $2 \mathrm{U} / \mathrm{ml}$ pyruvate kinase were added to the reaction buffer. The reaction was initiated by the addition of $10 \mu \mathrm{g}$ of mitochondria, and the changes in absorbance were recorded at $340 \mathrm{~nm}$ for $5 \mathrm{~min}$.

\section{Determination Of Intracellular ATP Levels}

The intracellular ATP concentrations were determined with the CellTiter-Glo Luminescent Cell Viability Assay. Cells were plated in 96-well plates at $2.5 \times 10^{4}$ cells/well, treated with $300 \mu \mathrm{M}$ palmitic acid (PA) for $24 \mathrm{~h}$, and then lysed with $100 \mu \mathrm{l}$ of lysis buffer. After the lysates were vortexed and centrifuged, the ATP contents were measured with a Glomax 96 Microplate Luminometer (Promega).

\section{Determination of MMP $\left(\Delta \Psi_{\mathrm{m}}\right)$ and MPT}

Membrane-permeable JC-1 dye has advantages over other cationic dyes in that it can selectively enter mitochondria and reversibly changes color (from green to red) as the membrane potential increases. In healthy cells with high $\Delta \Psi \mathrm{m}$, JC-1 spontaneously forms complexes with red fluorescence, whereas in unhealthy cells with low $\Delta \Psi \mathrm{m}$, JC-1 remains in the monomeric form, displaying only green fluorescence. Reduced fluorescence intensity indicates that the mitochondrial membrane structure is impaired. For the MPT assay, cells were treated with $1.0 \mu \mathrm{M}$ calcein-AM and $1.0 \mu \mathrm{M} \mathrm{CoCl}_{2}$ to selectively load the mitochondria with calcein by quenching the cytosolic calcein signal with $\mathrm{CoCl}_{2}$. The loss of mitochondrial calcein fluorescence under basal conditions was then used as a measure of nonpathological MPT.

\section{Assay Of SERCA Activity}

ER was extracted according to the manufacturer's instructions. In brief, after centrifugation at $600 \mathrm{~g}$ for $5 \mathrm{~min}$, the cells were harvested and then homogenized in the Isotonic Extraction Buffer using a Dounce homogenizer. After centrifugation at $1,000 \mathrm{~g}$ at $4{ }^{\circ} \mathrm{C}$ for $10 \mathrm{~min}$, the supernatant was collected, and centrifuged at $12000 \mathrm{~g}$ at $4^{\circ} \mathrm{C}$ for $15 \mathrm{~min}$. Subsequently, the supernatant was carefully transferred to a fresh tube and centrifuged at $100000 \mathrm{~g}$ at $4{ }^{\circ} \mathrm{C}$ for $60 \mathrm{~min}$. The pellet, which contained the ER compartment, was kept on ice before the experiments. SERCA activity was measured with a $\mathrm{Ca}^{2+}$-ATPase assay kit (Jiancheng Bioengineering Institute, Nanjing, China) and normalized to the protein concentration. This approach was the colorimetric measurement of Pi using the malachite green assay. An aliquot of extracted ER was added to the malachite green reagent and allowed to incubate at $37^{\circ} \mathrm{C}$ for $10 \mathrm{~min}$. The amount of Pi was determined at $636 \mathrm{~nm}$ against a standard curve. Pi generated during the reaction period was then converted to ATPase activity. 


\section{Preparation Of MAM Fractions From Cells And Mouse Livers}

The protocol described by Wieckowski et al ${ }^{26}$ was used to prepare the MAM from cultured cells and tissues. During each step of preparation, the mitochondrial and ER fractions were saved to assay their purity. The MAM fraction was finally collected and suspended in mitochondrion resuspension buffer (MRB) for further analysis. To prepare the MAM from liver tissues, all the procedures were similar to those described for the cellular preparation, except the tissue homogenization and centrifugation steps. The crude mitochondrial pellet was resuspended in IBliver-3 buffer and centrifuged at $10000 \mathrm{~g}$ for $10 \mathrm{~min}$ to isolate the MAM. The MAM fraction was suspended in mitochondrion resuspension buffer for further analysis.

\section{Western Blotting}

Protein concentrations were determined with a bicinchoninic acid protein quantitation kit. The aliquoted proteins $(100 \mu \mathrm{g})$ were separated with $12 \%$ SDS-PAGE, and after they were blotted onto membrane, primary antibodies were used to detect specific proteins. The primary antibodies were then detected with horseradish-peroxidase-labeled anti-IgG secondary antibodies. The blots were visualized with the Odyssey Two-Color Infrared Imaging System (Li-Cor, Lincoln, NE, USA).

\section{Calcium Measurement}

Cells $\left(1 \times 10^{6}\right)$ were washed with D-Hank's solution (Sigma) for three times and incubated at $37^{\circ} \mathrm{C}$ with Fura3-AM or Rhod-2-AM ( $1 \mu \mathrm{M}$; Molecular Probes) for $1 \mathrm{~h}$. Then the cells were washed with Hank's solution (Sigma) for 3 times and balanced at room temperature at least for $20 \mathrm{~min}$. The cells were then pretreated with BAPTA-AM $(100 \mu \mathrm{M})$ for $10 \mathrm{~min}$ to chelate the cytosolic $\mathrm{Ca}^{2+}$. The cells were then incubated with $\mathrm{PA}(300 \mu \mathrm{M})$ and the $\mathrm{Ca}^{2+}$ fluorescence intensity was measured with a Leica NT fluorescence confocal microscope (Leica, Wetzlar, Germany). To measure cytosolic $\mathrm{Ca}^{2+}$, the cells were treated with Fura3$\mathrm{AM}$ and their fluorescence (488 nm excitation; $515 \mathrm{~nm}$ emission) was recorded for $5 \mathrm{~min}$. To measure mitochondrial $\mathrm{Ca}^{2+}$, the cells were treated with Rhod-2AM and their fluorescence (559 nm excitation; $567 \mathrm{~nm}$ emission) was measured for $5 \mathrm{~min}$.

\section{Statistical Analysis}

Results are expressed as means \pm s.e. GraphPad (version 5.03, GraphPad Software Inc., CA, USA) was used to analyze the data. Comparisons of two groups were made with Student's $t$ test, and $P<0.05$ was considered statistically significant. All experiments were repeated at least twice.

\section{RESULTS}

HSS Attenuates Steatosis and Liver Injury in a Mouse Model of NASH

C57BL/6 mice that were fed an MCD diet for up to 2 weeks displayed clear manifestations of NASH and were used in the subsequent experiments. The bodyweights of the mice decreased after they were fed the MCD diet for 1 week $(P<0.05)$ or 2 weeks $(P<0.01)$, and the liver wet weights were reduced in the mice that were fed the MCD diet for 2 weeks $(P<0.01)$. The administration of Ad-HSS $\left(1 \times 10^{9}\right.$ plaque-forming units, $500 \mu \mathrm{l}$ ) to the MCD mice did not improve their bodyweights or liver wet weights compared with those of the Ad-Null group (Figure 1a and b).

The serum AST and ALT levels of the MCD mice increased after 1 or 2 weeks compared with those of the Ad-Null group $(P<0.05)$. The exogenous expression of Ad-HSS reduced the serum ALT levels in the MCD mice at 1 and 2 weeks $(P<0.05)$, but only reduced their serum AST levels at 2 weeks compared with those of the control (Ad-Null) group (Figure $1 c$ and $\mathrm{d}) ; P<0.05$. The serum TG levels increased in the MCD mice after 2 weeks compared with those in the control group $(P<0.01)$, but HSS transfection partly reduced the serum TG levels compared with those of the control mice $(P<0.05$; Figure 1e). The hepatic TG levels increased in the MCD mice after 1 and 2 weeks compared with those of the control group $(P<0.05$ and $P<0.01$, respectively). However, the exogenous expression of HSS reduced the hepatic TG levels in the MCD mice after 1 and 2 weeks $(P<0.05$ and $P<0.01$, respectively; Figure 1f).

A hepatic morphological analysis showed that the liver tissues of the MCD mice were filled with large numbers of fat vacuoles (Figure 1g). Oil red $\mathrm{O}$ staining showed that large amounts of lipid droplets were present in the hepatic tissues. The exogenous expression of HSS reduced the fat vacuoles and lipid accumulation within the liver tissues (Figure 1h), suggesting that HSS protects the liver from steatosis in mice with NASH.

To verify location and expressions of exogenously delivered HSS and their relation with attenuation of hepatic steatosis, the mice were injected with $500 \mu \mathrm{l}$ of the Ad-HSS $\left(1 \times 10^{7}, 10^{8}\right.$ and $10^{9}$ plaque-forming units) via tail vein and fed with an MCD diet for 2 weeks. It was found that exogenous HSS mainly localized in hepatocytes. More importantly, the exogenous HSS expression by adenoviral injection was apparently associated with attenuation of hepatic lipid deposition in a dose-related pattern $(P<0.05$; Figure 1i).

\section{HSS Suppresses ER Stress and Protects Mitochondria from Lipotoxicity}

After the mice were fed the MCD diet for 1 or 2 weeks, hepatic ER stress, manifesting as the elevated expression of GRP78 and CHOP, was induced and HSS expression was downregulated. However, the exogenous expression of HSS inhibited this ER stress (Figure 2a), and cell apoptosis, detected as the elevated expression of BAX and BCL-2, also 

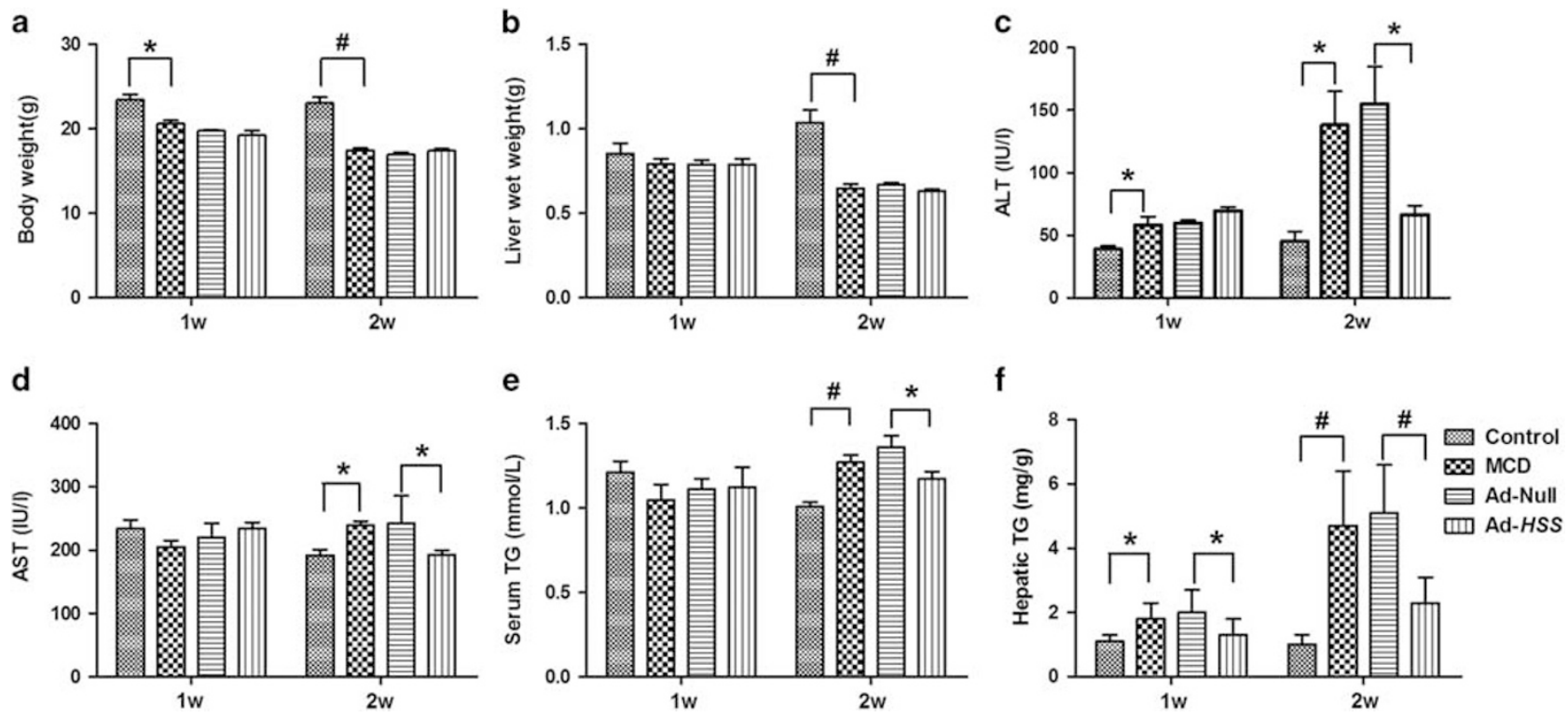

Figure 1 Hepatic stimulator substance (HSS) attenuates steatotic injury. C57BL/6 mice were fed an methionine and choline-deficient (MCD) diet for 2 weeks to establish a mouse model of nonalcoholic steatohepatitis (NASH). (a) Bodyweight. (b) Liver wet weight. (c) Serum alanine aminotransferase (ALT). (d) Serum aspartate aminotransferase (AST). (e) Serum triglyceride (TG). (f) Hepatic TG. (g) Liver tissues stained with hematoxylin and eosin. (h) Liver tissues stained with oil red O. (i) Topographical relationship between steatosis area and HSS expression. Data are presented as mean \pm s.e. ( $n=12$ per group). ${ }^{*} P<0.05,{ }^{\#} P<0.01$.

decreased (Figure 2b). The exogenous expression of HSS effectively reduced liver apoptosis levels in the MCD mice, suggesting that HSS protects the mitochondria from lipidinduced apoptosis.

\section{HSS Enhances SERCA Expression in MAMs of Mice with NASH}

SERCA is an ER-associated calcium pump, responsible for the transport of $\mathrm{Ca}^{2+}$ from the cytosol to the ER, helping to maintain the intracellular $\mathrm{Ca}^{2+}$ concentrations at physiological levels. To study the mechanism underlying the protective effects of HSS in terms of SERCA, the MAM fractions were extracted from the livers of mice with $\mathrm{NASH}$, and the liver cells were subjected to lipid intoxication. Western blotting results indicated that hepatic SERCA expression was reduced in the MCD mice relative to that in the control mice. However, SERCA expression in the mice expressing exogenous HSS was restored to levels similar to those of the control mice, indicating that HSS maintains the expression of SERCA from ER stress (Figure 3). Furthermore, the protective effects of HSS on SERCA occurred in both the ER and MAM compartments (Figure 3). These results clearly indicate that the exogenous expression of the HSS gene increases SERCA expression in the MAM compartment.

\section{Inhibition of ER Stress by HSS Attenuates Steatosis}

To determine whether the increased SERCA activity induced by the exogenous expression of HSS improves hepatocyte resistance to lipid injury, cells were treated with PA $(300 \mu \mathrm{M})$ for $24 \mathrm{~h}$. A western blotting analysis indicated that the PA treatment induced ER stress, based on the expression and upregulation of several ER-stress-related markers, such as GRP78 and CHOP. ER stress was alleviated in the HSS-Tr/ 7402 cells (Figure 4a). To convince our results, another cell line HepG2 was employed in the experiment. The results were identical to those of BEL-7402 cells, indicating that ER stress was decreased in the HSS-Tr/HepG2 cells (Figure 4a). The PA-induced collapse of the mitochondrial membrane structure (ie, impaired MPT and MMP) was also prevented in the HSS-Tr/7402 cells (Figure $4 \mathrm{~b}$ and c), resulting in improved respiratory chain complex $\mathrm{V}$ activity and ATP levels relative to those in the BEL-7402 cells transfected with vector-Tr (Figure $4 \mathrm{~d}$ and e). The TG levels were also reduced in the HSS-Tr/ 7402 cells (Figure 4f). In the vector-Tr/7402 cells, PA treatment caused morphological abnormalities in the mitochondrial ultrastructure, including the loss of mitochondria, the swelling and vacuolization of the organelle, and swollen/ deficient cristae. However, in the HSS-expressing/7402 cells, the structure of the mitochondria was markedly improved, with abundant mitochondria and healthy cristae, and dilatation of the cristae structure was rarely observed. (Figure 4g). Like the vector- $\operatorname{Tr} / 7402$ cells, PA treatment could induce the abnormalities of mitochondrial ultrastructure in vector- $\mathrm{Tr} / \mathrm{HepG} 2$ cells. However, the transfection of HSS could improve the structure of the mitochondria (Figure 4g).

\section{HSS Rescues SERCA Expression to Inhibit ER Stress}

The reduced SERCA expression induced by PA $(300 \mu \mathrm{M})$ was slightly ameliorated in the HSS-Tr/7402 cells compared with 
g
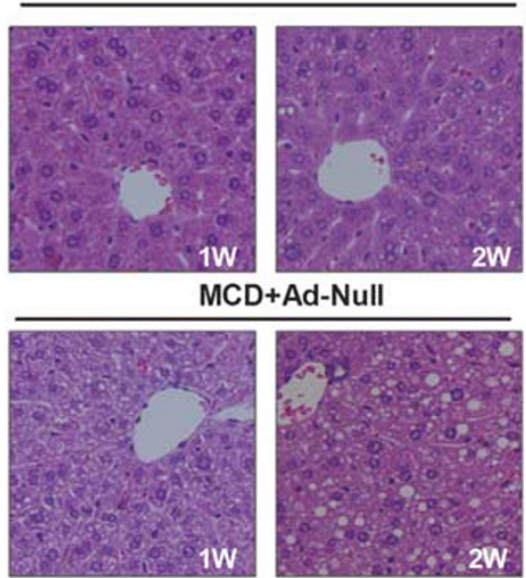

h

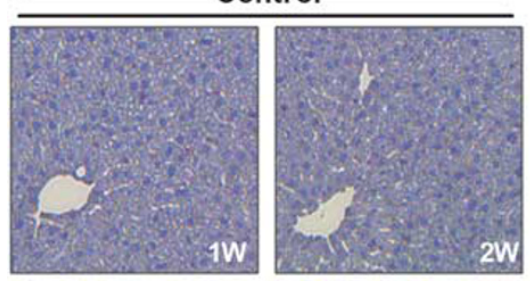

MCD+Ad-Null
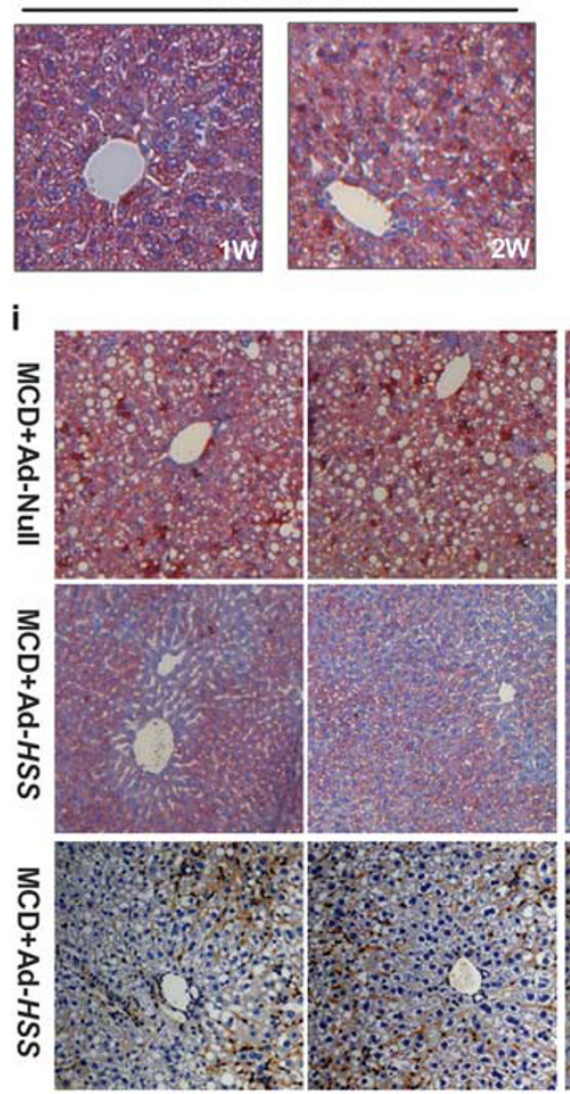

$10^{7}$ $10^{8}$

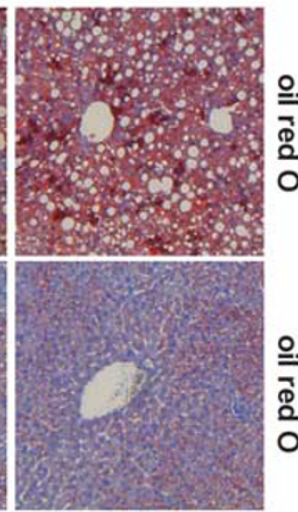

$$
\begin{aligned}
& \frac{0}{7} \\
& \frac{0}{0} \\
& 0
\end{aligned}
$$

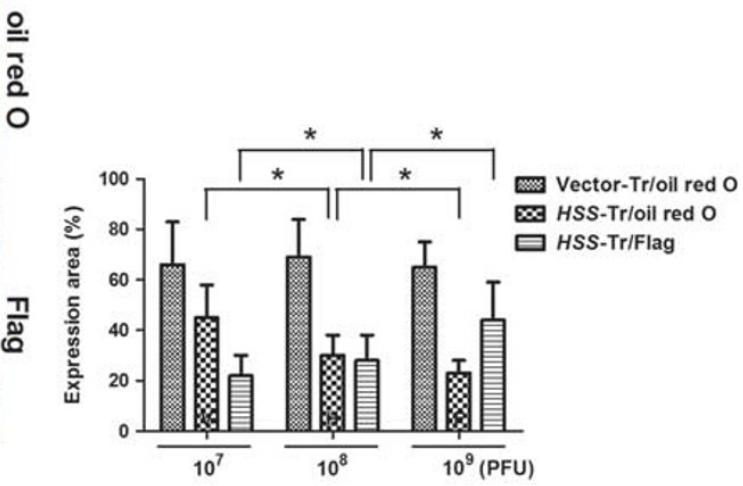

Figure 1 Continued 

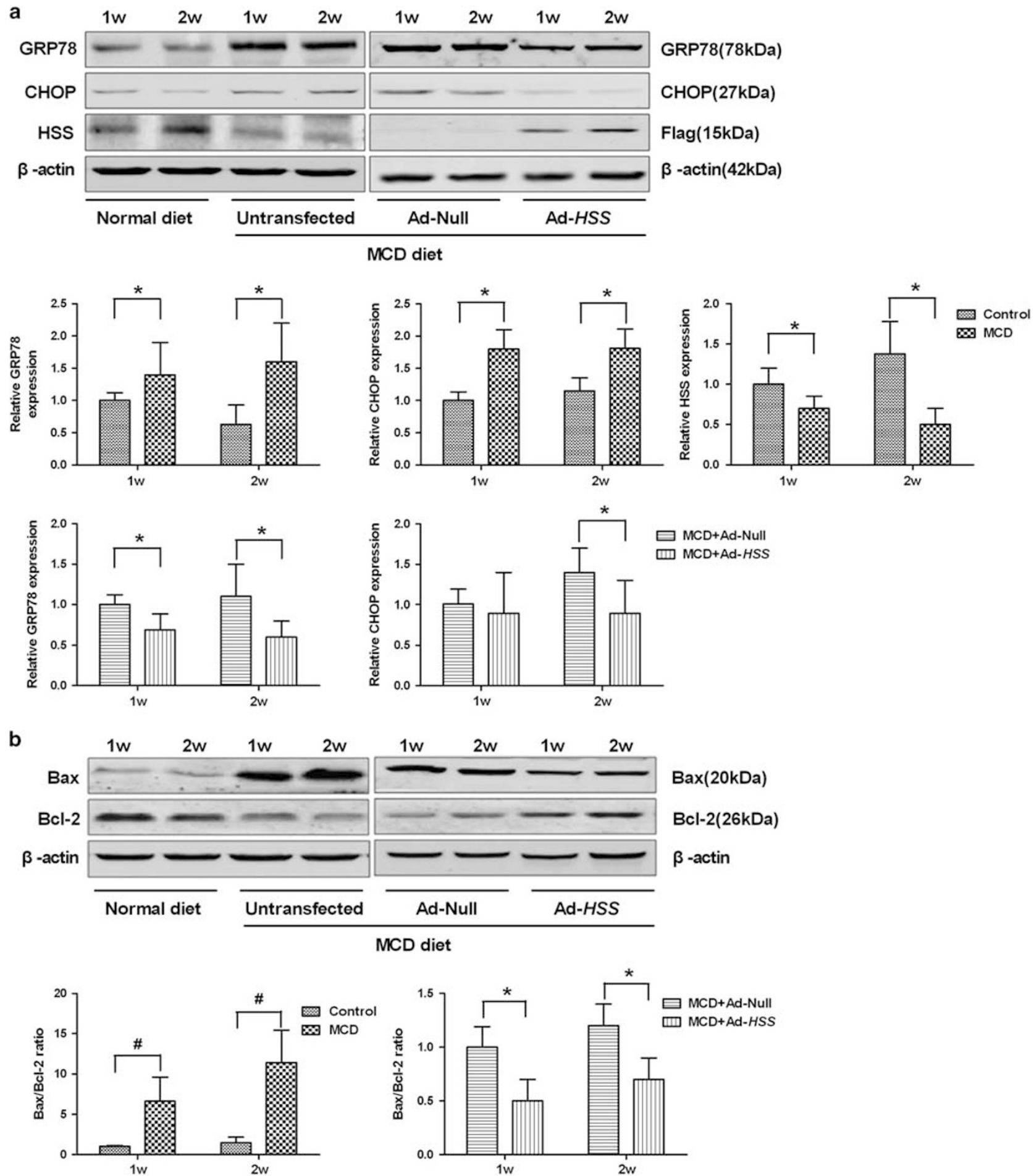

$\operatorname{Bax}(20 \mathrm{kDa})$

$\mathrm{Bcl}-2(26 \mathrm{kDa})$

$\beta$-actin

MCD diet

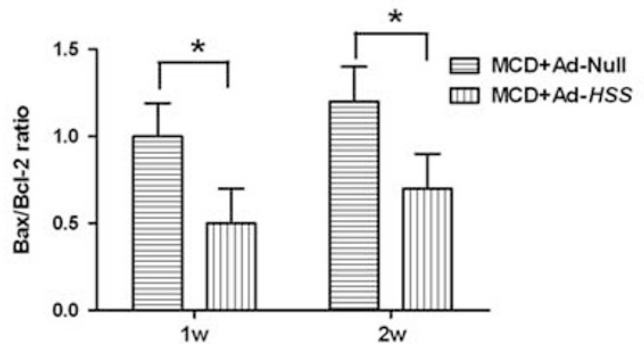

Figure 2 Hepatic stimulator substance (HSS) suppresses endoplasmic reticulum (ER) stress and mitochondrial apoptosis in mice with nonalcoholic steatohepatitis (NASH). (a) Levels of ER stress markers and HSS were determined with western blotting and normalized to $\beta$-actin. (b) Levels of apoptotic indices BAX and BCL-2 were determined with western blotting and normalized to $\beta$-actin. Densitometry results are shown. Data are presented as mean \pm s.e. ( $n=3$ per group). ${ }^{*} P<0.05,{ }^{\#} P<0.01$.

that in the vector- $T r / 7402$ cells (Figure $5 \mathrm{a}$ ). We also used the HSS-Tr/HepG2 cells to convince the results above. As we expected, the decreased SERCA expression promoted by PA was also inhibited in the HSS-Tr/HepG2 cells (Figure 5a). SERCA activity was also preserved in the HSS-Tr/7402 cells (Figure 5b). Moreover, SERCA rescue occurred not only in 
a
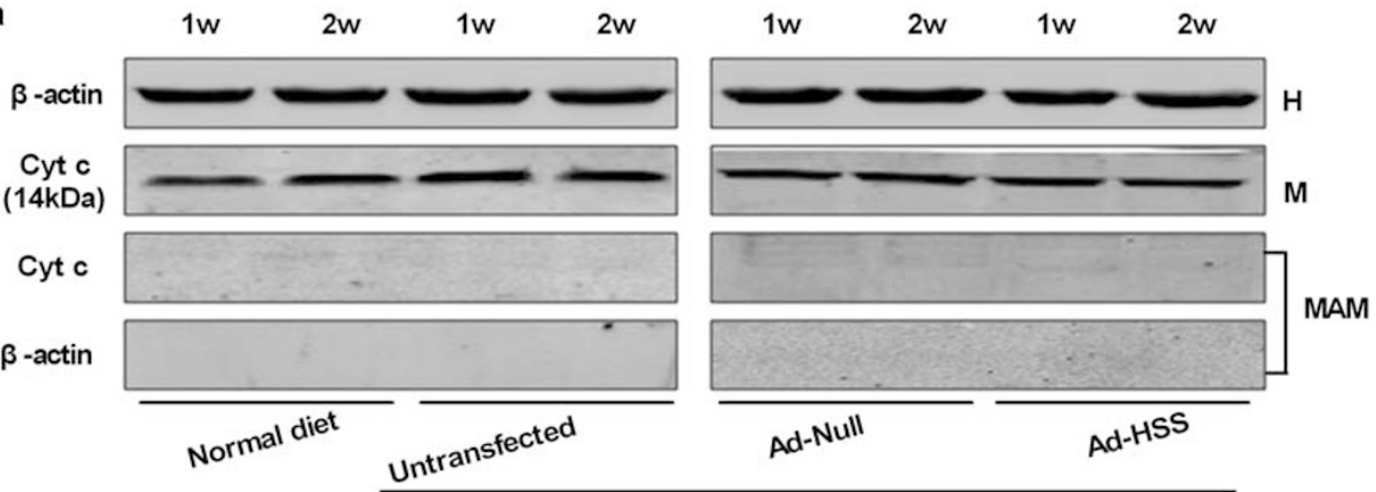

\section{MCD diet}

b
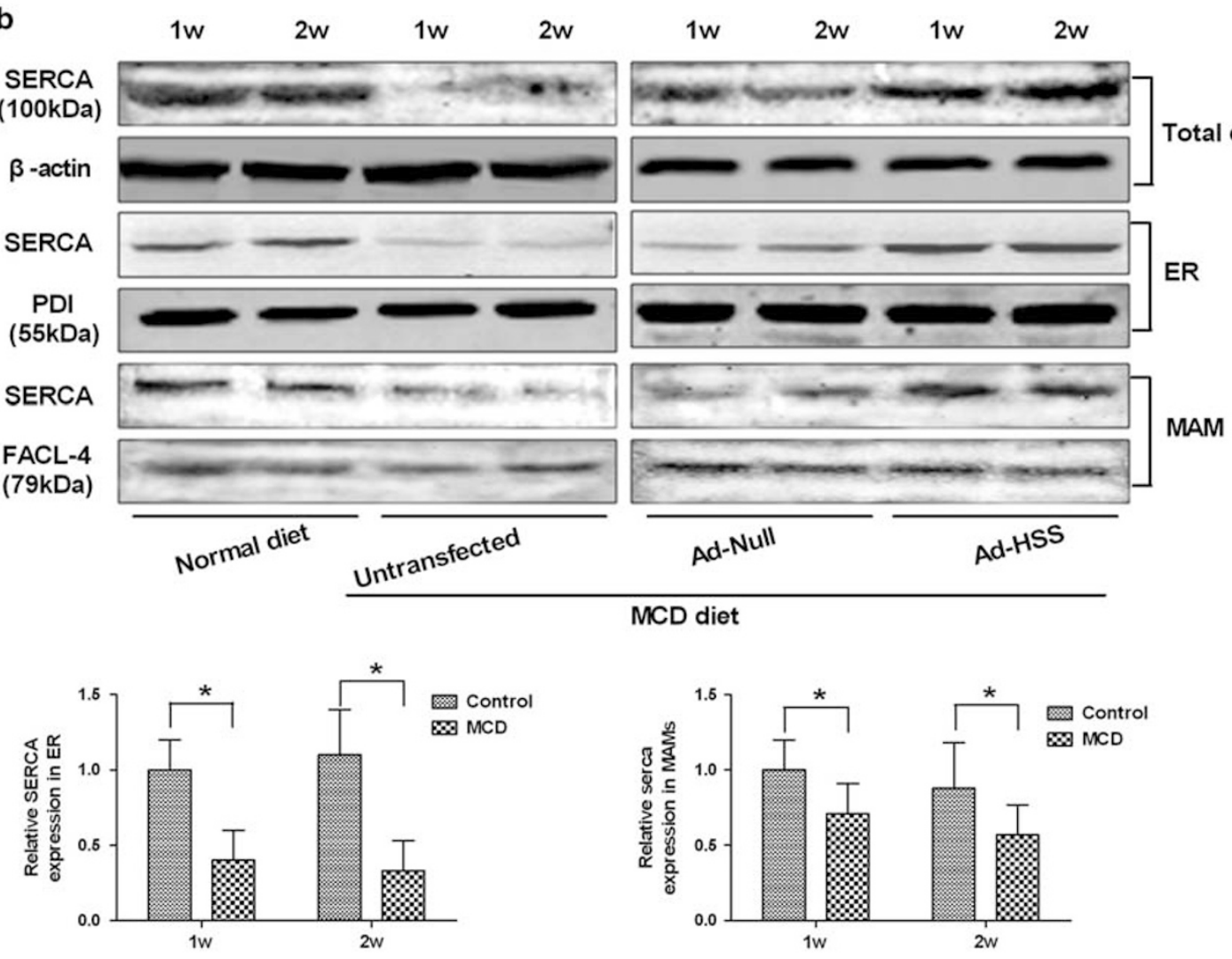

\section{MCD diet}
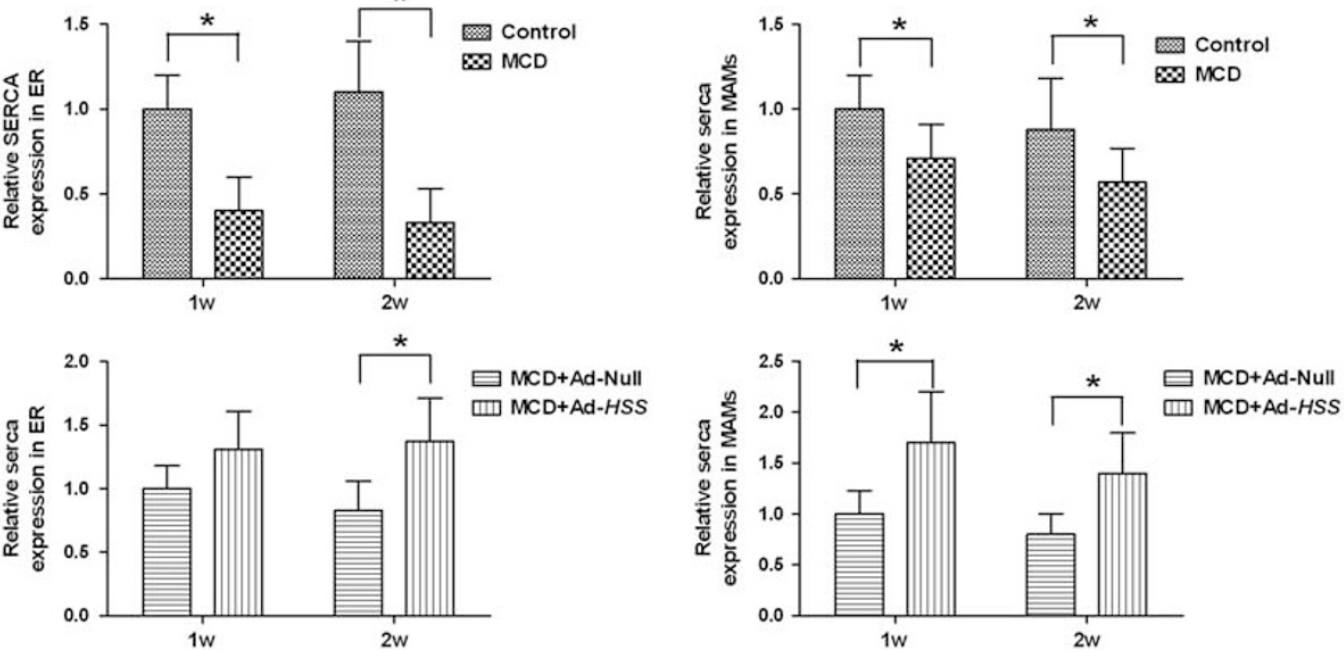

Figure 3 Hepatic stimulator substance (HSS) enhances sarco/ER calcium ATPase (SERCA) expression in mitochondria-associated membrane (MAM) in mice with nonalcoholic steatohepatitis (NASH). Proteins were extracted from the cell cytosol, endoplasmic reticulum (ER), and MAM. Western blotting was used to determine the purity of MAM (a) SERCA expression in the cytosolic, ER, and MAM extracts (b). As an ER marker, protein disulfide isomerase (PDI) should be enriched in the ER fraction, and as a MAM marker, FACL-4 should also be enriched in this fraction. Cytochrome $c$ localizes to the inner mitochondrial membrane and should be heavily expressed in the mitochondrial fraction, but not in the MAM fraction. $\beta$-actin should be absent from the mitochondrial, MAM, and ER compartments. Densitometry results are shown. Data are presented as mean \pm s.e. ( $n=3$ per group). ${ }^{*} P<0.05$. 
a
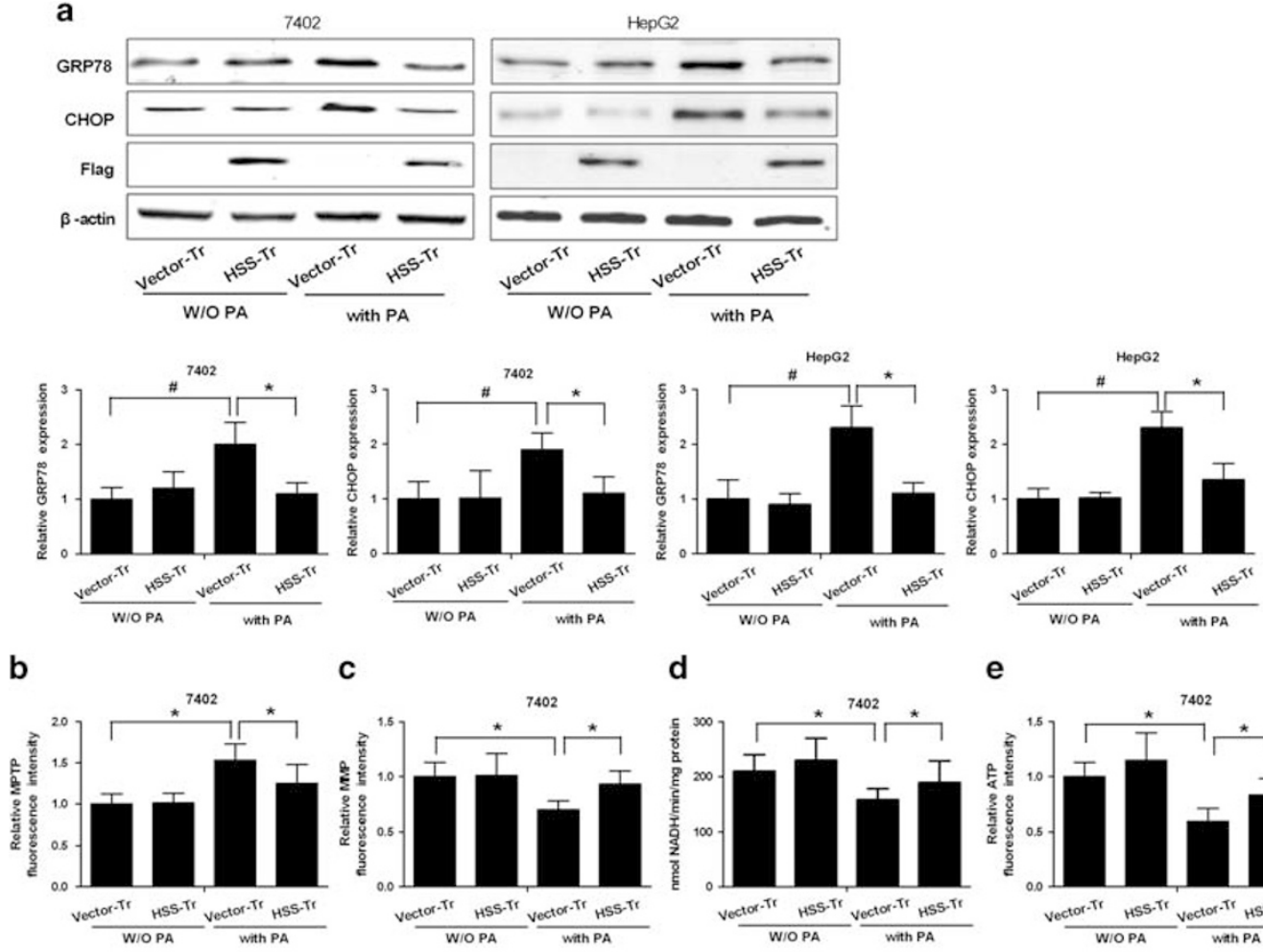

c

d

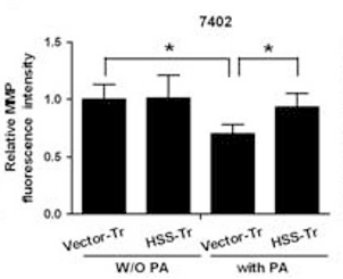

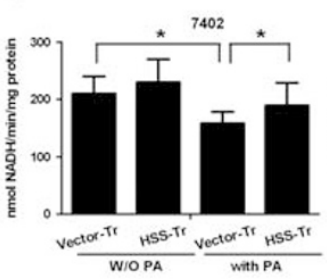

e

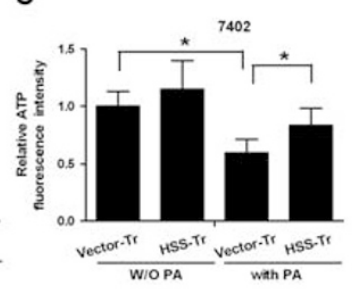

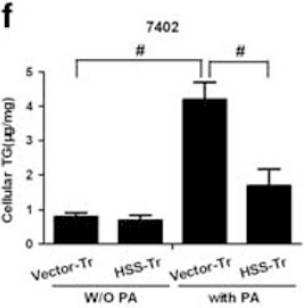

g
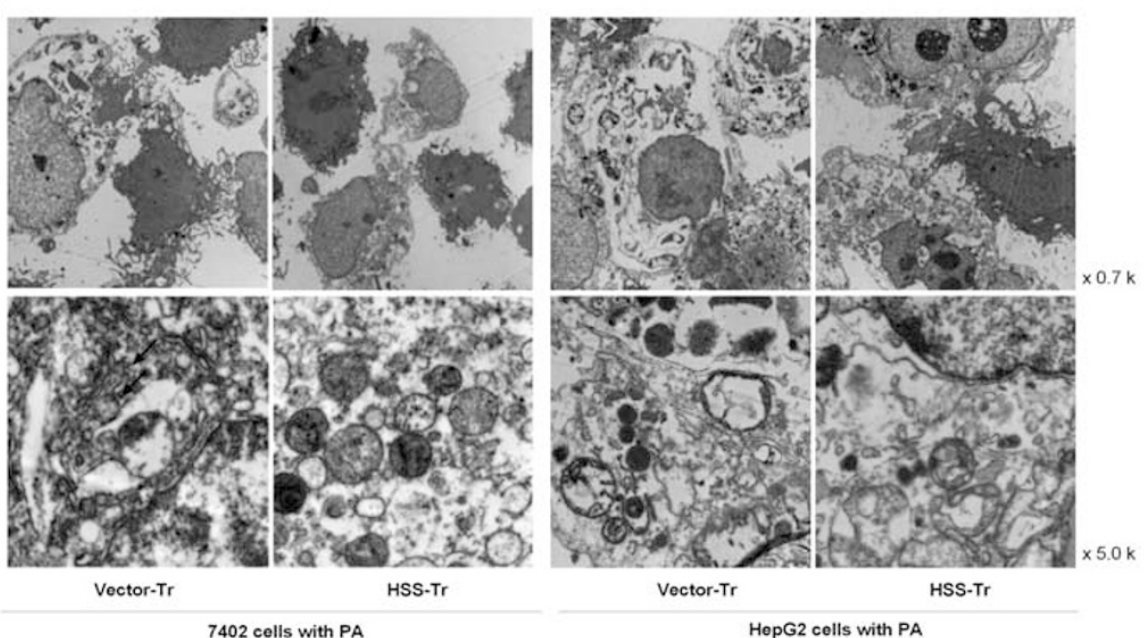

Figure 4 Hepatic stimulator substance (HSS) expression inhibits endoplasmic reticulum (ER) stress and attenuates mitochondrial damage. Cells were treated with palmitic acid $(\mathrm{PA} ; 300 \mu \mathrm{M})$ for $24 \mathrm{~h}$. (a) Levels of ER stress markers were determined in the HSS-transfected (HSS-Tr) and control cells with western blotting. Densitometry results are shown. Relative MTP $(\mathbf{b})$ and MMP (c) fluorescence intensities; mitochondrial complex V activity (d); relative ATP contents (e); and cellular TG levels (f). (g) Electron microscopic image of mitochondria. Palmitic acid (PA) treatment caused marked disintegration of the mitochondrial ultrastructure, including the loss in mitochondria, mitochondrial swelling, and vacuolization) in most hepatocytes. HSS transfection markedly attenuated the morphological injuries to the mitochondria induced by PA. Data are presented as mean \pm s.e. $\left(n=3\right.$ per group). ${ }^{*} P<0.05$, $\# P<0.01$.

the ER membrane, but also in the MAM compartment in the HSS-Tr/7402 cells (Figure 5c).

\section{HSS Protects Mitochondria from $\mathrm{Ca}^{2+}$ Overload}

It has been reported that the mitochondrial $\mathrm{Ca}^{2+}$ uptake from the cytosol requires the direct contact between the mitochondria and the ER membrane via the MAM. Therefore, we investigated whether the protective effect of HSS on SERCA expression during ER stress protects the mitochondria from $\mathrm{Ca}^{2+}$ overflow via the MAM compartment. To address this question, ER stress was induced with PA, and the cytosolic and mitochondrial $\mathrm{Ca}^{2+}$ oscillations were monitored after HSS transfection. The results indicate that the release of $\mathrm{Ca}^{2+}$ into the cytosol and mitochondria increased immediately 
a
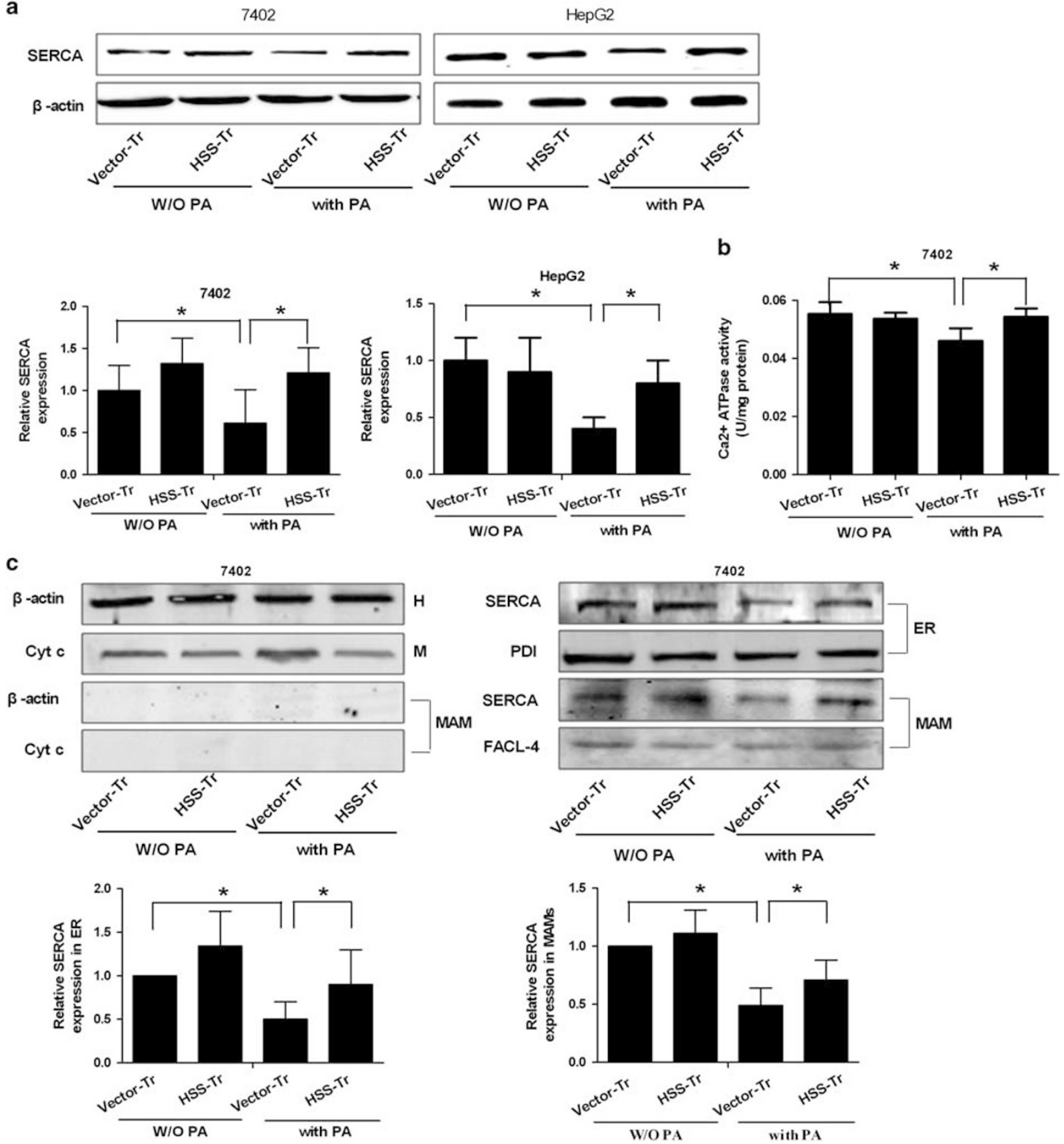

Figure 5 HSS promotes SERCA expression at MAM. Cells were treated with PA (300 $\mu$ M) for 24 h. (a) Levels of SERCA were determined with western blotting. Densitometry results are shown. (b) SERCA activity was analyzed. (c) SERCA expression was measured in the ER and MAM compartments. Densitometry results are shown. ${ }^{*} P<0.05$.

after the addition of PA to vector-Tr cells, whereas the $\mathrm{Ca}^{2+}$ levels in these two compartments decreased in the HSS-Tr cells (Figure 6). As it is hard to measure SERCA activity in the MAM compartment, we used the calcium-specific chelator BAPTA-AM $(100 \mu \mathrm{M})$ for $10 \mathrm{~min}$ to abolish the oscillations in free cytosolic $\mathrm{Ca}^{2+}$. This strategy excluded any possibility that the free intracellular $\mathrm{Ca}^{2+}$ would move into the mitochondria, except through the MAM compartment. Under these conditions, the mitochondrial $\mathrm{Ca}^{2+}$ levels are partly dependent on the SERCA activity in the MAM. As shown in Figure 6a, the PA-induced increase in $\mathrm{Ca}^{2+}$ release was abolished in the cytosolic compartment. A reduction in the mitochondrial $\mathrm{Ca}^{2+}$ concentration was also observed in the BAPTA-AM-pretreated cells (Figure 6b), suggesting that ER stress induces an increase in cytosolic $\mathrm{Ca}^{2+}$ as a result of the inactivation of SERCA, permitting the influx of free $\mathrm{Ca}^{2+}$ into 
a

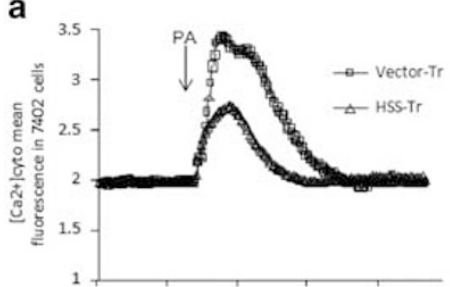

b

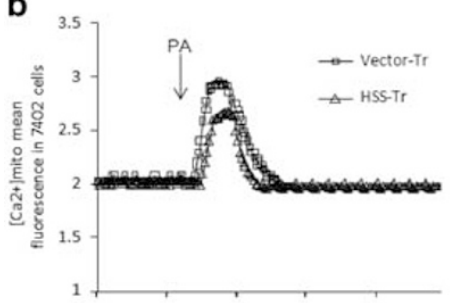

C
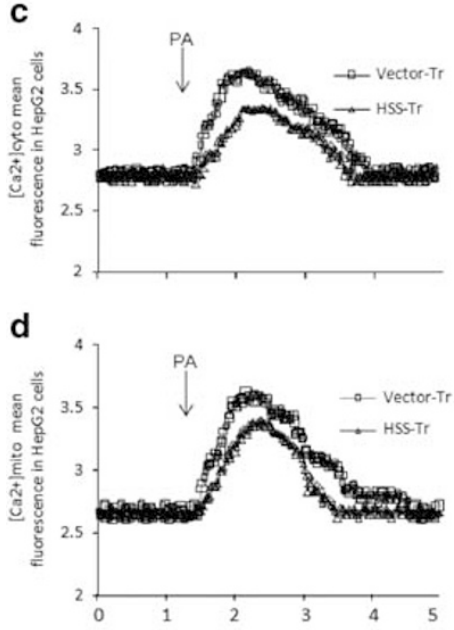
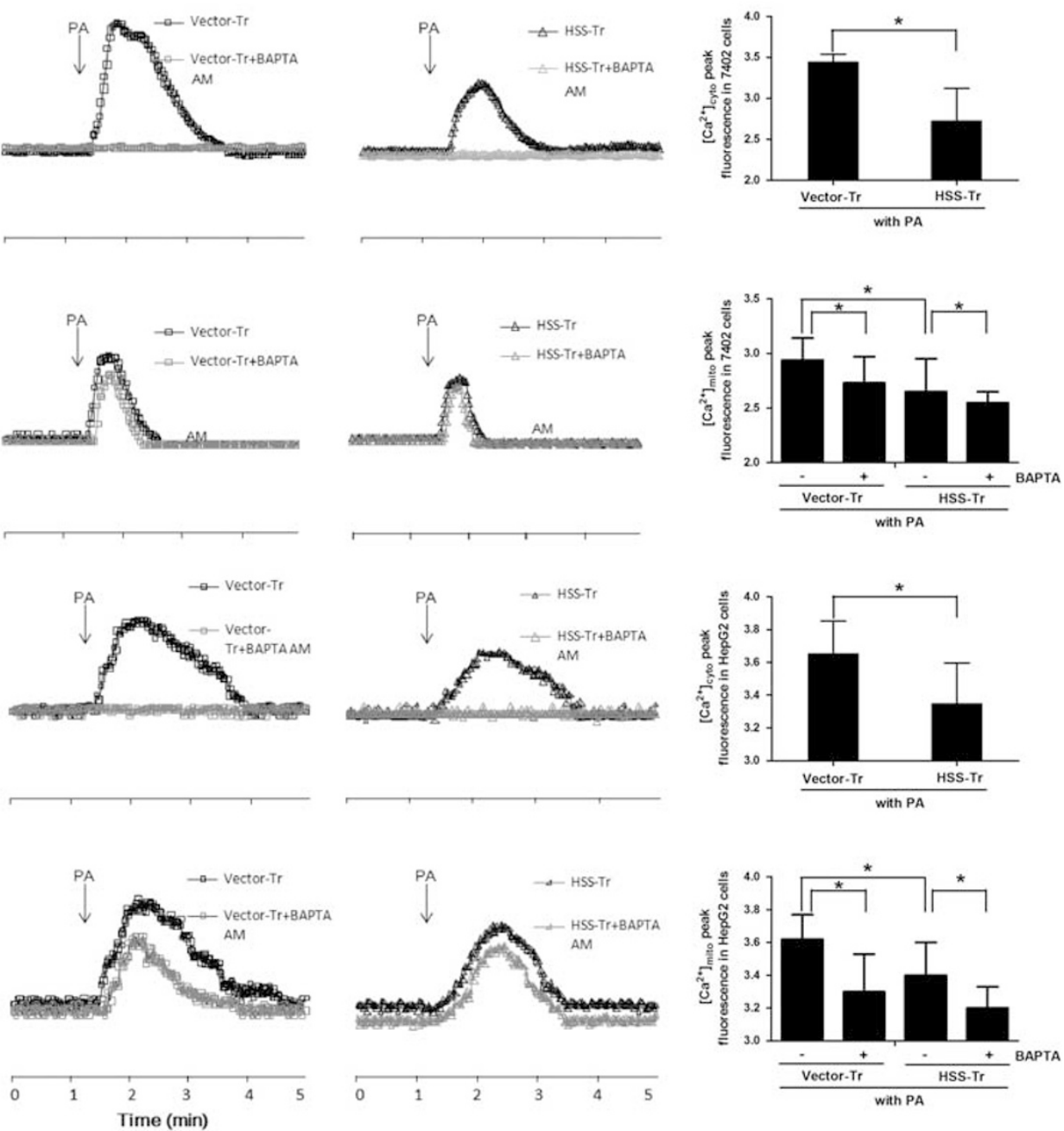

Figure 6 Hepatic stimulator substance (HSS) protects sarco/ER calcium ATPase (SERCA) in the MAM compartment to maintain intracellular Ca ${ }^{2+}$ homeostasis. $\mathrm{Ca}^{2+}$ fluorescence intensity was measured with confocal microscopy. Cells were stimulated with palmitic acid (PA; $\left.300 \mu \mathrm{M}\right)$. Fluorescence results of (a) cytosolic and (b) mitochondrial $\mathrm{Ca}^{2+}$ in 7402 cells. Fluorescence results of (c) cytosolic and (d) mitochondrial $\mathrm{Ca}^{2+}$ in $\mathrm{HepG}^{2}$ cells.

the mitochondria through the MAM compartment. Consequently, mitochondrial $\mathrm{Ca}^{2+}$ levels increased sharply after PA treatment. However, the mitochondrial $\mathrm{Ca}^{2+}$ levels were lower in the HSS-Tr cells than in the control (vector-Tr) cells, suggesting that HSS protects SERCA in the MAM compartment (Figure 6b). The above results were also convinced in the HepG2 cells (Figure $6 \mathrm{c}$ and d).

\section{DISCUSSION}

As mentioned above, ER stress is one of the major contributors to the pathogenesis of NASH. As a primary regulator of $\mathrm{Ca}^{2+}$, SERCA2b inhibits the sustained release of $\mathrm{Ca}^{2+}$ through the ER lumen, maintains the homeostasis of intracellular calcium, and alleviates ER stress. Therefore, increased SERCA2b concentrations have been used as pilot treatments of type II diabetes and NASH. ${ }^{27}$ Supporting this use, we have confirmed that the exogenous expression of HSS attenuated hepatic steatosis by inhibiting ER stress and preserving the mitochondrial functions, which appeared to be associated with the regulation of SERCA activity.

Steatosis is accompanied by increasing ROS generation, which may lead to further liver damage. ${ }^{28}$ The mitochondrial respiratory chain is the primary source of ROS, which can damage the mitochondrial DNA, proteins, and lipids. ${ }^{29}$ Altering the ROS levels occurred under ER stress can also affect the intracellular redox status and calcium homostasis, which has a critical role in the regulation of SERCA activity by promoting multiple redox-dependent modifications of SERCA Cys674. ${ }^{30}$ SERCA is a $\mathrm{Ca}^{2+}$ ATPase that is responsible for transferring $\mathrm{Ca}^{2+}$ from the cytosol into the ER lumen at the expense of ATP hydrolysis. Three isoforms (SERCA1-3) have been identified and are encoded by three different genes (ATP2A1-3) in mammals. SERCA2b is the primary isoform of SERCA2 and predominantly occurs in the liver, ${ }^{31}$ where it is an important regulator of ER stress and lipid homeostasis. The inhibition of SERCA activity can result in the release of 
$\mathrm{Ca}^{2+}$ from the ER lumen and the disruption of ER homeostasis. ${ }^{32}$

Our previous study also demonstrated that HSS reduces ROS concentrations, protecting SERCA activity. Here, we demonstrated that NASH is accompanied by reduced SERCA expression in the MAM compartment and the onset of mitochondrial $\mathrm{Ca}^{2+}$ overload. Simultaneously with the inactivation of SERCA by PA, the HSS content also decreased, indicating that the depletion of HSS protein correlated with the progression of ER stress. Furthermore, when HSS was introduced at this time, SERCA expression was restored and ER stress was alleviated. Therefore, we propose that the HSSinduced inhibition of ER stress is attributable to the preservation of SERCA expression by HSS, particularly in the MAM compartment, during free-fatty-acid-induced lipotoxicity.

MAM is a structure conserved across all eukaryotic phyla, and is a key determinant of cell survival or death by regulating the transfer of $\mathrm{Ca}^{2+}$ and other metabolites. SERCA, a MAMenriched protein, ${ }^{33}$ is responsible for pumping cytoplasmic $\mathrm{Ca}^{2+}$ back into the ER lumen. The proper maintenance of the MAM and the $\mathrm{Ca}^{2+}$ flux is essential for mitochondrial function and metabolic homeostasis. ${ }^{34}$ During ER stress, the extent of the MAM increases and the ER and mitochondria move closer together, probably causing a change in the MAM proteome. ${ }^{35}$ The integrity of the ER/mitochondrial $\mathrm{Ca}^{2+}$ cross-talk is impaired under ER stress, possibly by variations in the extent and composition of the MAM. In our experiment, the expression of SERCA in the MAM region was reduced during ER stress, weakening the cytoplasmic $\mathrm{Ca}^{2+}$ influx and particularly the MAM transport of $\mathrm{Ca}^{2+}$ into the ER lumen.

The depletion of ER calcium inhibits oxidative protein folding, which may be attributed to another key regulator of calcium homeostasis and signaling, for example, calnexin. Calnexin depalmitoylation during ER stress impairs the association between calnexin and SERCA2b, ${ }^{36}$ and the blockade of the interaction between calnexin and SERCA could inhibit SERCA activity and accelerate the mitochondrial $\mathrm{Ca}^{2+}$ overload. Therefore, the ER chaperones and oxidoreductases in the MAM compartment should be screened for potential interactive partner with SERCA to determine the extent to which they regulate calcium homeostasis.

Aberrant $\mathrm{Ca}^{2+}$ regulation in the ER is a primary cause of hepatic ER dysfunction and stress. ${ }^{37}$ Altered $\mathrm{Ca}^{2+}$ signaling through the MAM plays a key role in connecting ER stress to mitochondrial dysfunction. The proportion of the ER in close contact with the mitochondria compared with the total ER content is higher in the livers of high-fat-diet mice than in those of the controls. ${ }^{38}$ In the present study, we demonstrated that SERCA expression in the MAM regulated the calcium homeostasis between the ER and the mitochondria. We speculate that an important reason for this is that SERCAregulated $\mathrm{ER}$ stress causes the $\mathrm{ER}$ and mitochondria to move closer together, resulting in more rapid $\mathrm{Ca}^{2+}$ transfer from the
ER to the mitochondria through the MAM. Higher rates of $\mathrm{Ca}^{2+}$ accumulation in the mitochondria impair the mitochondrial oxidative capacity, increase ROS generation, and cause cellular stress and abnormal metabolism.

Thus far, the contribution of the MAM-localized protein SERCA to the development of NASH remains unclear. This study provides the first demonstration of the protective effect of HSS on SERCA expression in the MAM during lipotoxic injury, and shows that this protection reduces ER stress and helps to maintain the intracellular $\mathrm{Ca}^{2+}$ concentrations at physiological levels, thus effectively preventing hepatic steatosis. These results provide novel insight into the interactions between HSS and SERCA, and their potential application in the treatment of NASH.

\section{ACKNOWLEDGMENTS}

This work was supported by the National Natural Science Foundation of China (Grant No. 31371169).

\section{DISCLOSURE/CONFLICT OF INTEREST}

The authors declare no conflict of interest.

1. Neuschwander-Tetri BA, Caldwell SH. Nonalcoholic steatohepatitis: summary of an AASLD single topic conference. Hepatology 2003;37: 1202-1219.

2. Fan JG, Farrell GC. Epidemiology of non-alcoholic fatty liver disease in China. J Hepatol 2009;50:204-210.

3. Puri $\mathrm{P}$, Mirshahi F, Cheung $\mathrm{O}$, et al. Activation and dysregulation of the unfolded protein response in nonalcoholic fatty liver disease. Gastroenterology 2008;134:568-576.

4. Dara L, Ji C, Kaplowitz N. The contribution of endoplasmic reticulum stress to liver diseases. Hepatology 2011;53:1752-1763.

5. Rusiñol $A E$, Cui $Z$, Chen $M H$, et al. A unique mitochondria-associated membrane fraction from rat liver has a high capacity for lipid synthesis and contains pre-Golgi secretory proteins including nascent lipoproteins. J Biol Chem 1994;269:27494-27502.

6. Patergnani S, Suski JM, Agnoletto C, et al. Calcium signaling around Mitochondria Associated Membranes (MAMs). Cell Commun Signal 2011;9:19.

7. Rizzuto $\mathrm{R}$, Marchi $\mathrm{S}$, Bonora $\mathrm{M}$, et al. $\mathrm{Ca}(2+)$ transfer from the $\mathrm{ER}$ to mitochondria: when, how and why. Biochim Biophys Acta 2009;1787: 1342-1351.

8. LaBrecque DR, Pesch LA. Preparation and partial characterization of hepatic regenerative stimulator substance (SS) from rat liver. J physiol 1975;248:273-284.

9. Kondili VG, Tzirogiannis KN, Androutsos CD, et al. The hepatoprotective effect of hepatic stimulator substance (HSS) against liver regeneration arrest induced by acute ethanol intoxication. Dig Dis Sci 2005;50: 297-307.

10. LaBrecque DR, Bachur NR. Hepatic stimulator substance: physicochemical characteristics and specificity. Am J Physiol 1982;242:G281-G288.

11. Mei $\mathrm{MH}, \mathrm{An} \mathrm{W}$, Zhang $\mathrm{BH}$, et al. Hepatic stimulator substance protects against acute liver failure induced by carbon tetrachloride poisoning in mice. Hepatology 1993;17:638-644.

12. Li S, Tang Z, Yu H, et al. Administration of naked plasmid encoding hepatic stimulator substance by hydrodynamic tail vein injection protects mice from hepatic failure by suppressing the mitochondria permeability transition. J Pharmacol Exp Ther 2011;338:750-757.

13. Wu Y, Chen $\mathrm{L}, \mathrm{Yu} \mathrm{H}$, et al. Transfection of hepatic stimulator substance gene desensitizes hepatoma cells to $\mathrm{H} 2 \mathrm{O} 2$-induced cell apoptosis via preservation of mitochondria. Arch Biochem Biophys 2007;464:48-56.

14. Jiang SJ, Li W, An W. Adenoviral gene transfer of hepatic stimulator substance confers resistance against hepatic ischemia-reperfusion injury by improving mitochondria function. Hum Gene Ther 2013;24:443-456. 
15. Wu Y, Zhang J, Dong L, et al. Hepatic stimulator substance mitigates hepatic cell injury through suppression of the mitochondria permeability transition. FEBS J 2010;277:1297-1309.

16. Jiang $Y$, Zhao M. An W. Increased hepatic apoptosis in high-fat diet-induced NASH in rats may be associated with downregulation of hepatic stimulator substance. J Mol Med (Berl) 2011;89:1207-1217.

17. Francavilla $A$, Hagiya $M$, Porter $K A$, et al. Augmenter of liver regeneration: its place in the universe of hepatic growth factors. Hepatology 1994;20:747-757.

18. Li W, Zhang J, An W. The conserved CXXC motif of hepatic stimulator substance is essential for its role in mitochondria protection in $\mathrm{H}_{2} \mathrm{O} 2-$ induced cell apoptosis. FEBS Lett 2010;584:3929-3935.

19. Zhang J, Li Y, Jiang S, et al. Enhanced endoplasmic reticulum SERCA activity by overexpression of hepatic stimulator substance gene prevents hepatic cells from ER stress-induced apoptosis. Am J Physiol Cell Physiol 2014;306:C279-C290.

20. Wu CK, Dailey TA, Dailey HA, et al. The crystal structure of augmenter of liver regeneration: A mammalian FAD-dependent sulfhydryl oxidase. Protein Sci 2003;12:1109-1118.

21. Lisowsky $\mathrm{T}$, Weinstat-Saslow DL, Barton $\mathrm{N}$, et al. A new human gene located in the PKD1 region of chromosome 16 is a functional homologue to ERV1 of yeast. Genomics 1995;29:690-697.

22. Giorda R, Hagiya $M$, Seki $T$, et al. Analysis of the structure and expression of the augmenter of liver regeneration (ALR) gene. Mol Med 1996;2:97-108.

23. Banci L, Bertini I, Cefaro C, et al. An intrinsically disordered domain has a dual function coupled to compartment-dependent redox control. J Mol Biol 2013:425:594-608.

24. Allen S, Balabanidou V, Sideris DP, et al. Erv1 mediates the Mia40dependent protein import pathway and provides a functional link to the respiratory chain by shuttling electrons to cytochrome c.J Mol Biol 2005;353:937-944.

25. Thirunavukkarasu C, Wang LF, Harvey SA, et al. Augmenter of liver regeneration: an important intracellular survival factor for hepatocytes. J Hepatology 2008;48:578-588.

26. Wieckowski MR, GiorgiC, Lebiedzinska $M$, et al. Isolation of mitochondria-associated membranes and mitochondria from animal tissues and cells. Nat Protoc 2009;4:1582-1590.
27. Park SW, Zhou Y, Lee J, et al. Sarco (endo) plasmic reticulum Ca2 + -ATPase $2 b$ is a major regulator of endoplasmic reticulum stress and glucose homeostasis in obesity. Proc Natl Acad Sci USA 2010;107: 19320-19325.

28. Cardoso AR, Kakimoto PA, Kowaltowski AJ. Diet-sensitive sources of reactive oxygen species in liver mitochondria: role of very long chain acyl-CoA dehydrogenases. PLoS One 2013;8:e77088.

29. Paradies G, Paradies V, Ruggiero FM, et al. Oxidative stress, cardiolipin and mitochondrial dysfunction in nonalcoholic fatty liver disease. World J Gastroenterol 2014:20:14205-14218.

30. Lancel S, Zhang J, Evangelista A, et al. Nitroxyl activates SERCA in cardiac myocytes via glutathiolation of cysteine 674. Circ Res 2009;104: 720-723.

31. Vangheluwe $P$, Raeymaekers $L$, Dode $L$, et al. Modulating sarco (endo) plasmic reticulum Ca2+ ATPase 2 (SERCA2) activity: cell biological implications. Cell Calcium 2005;38:291-302.

32. Cardozo AK, Ortis F, Storling J, et al. Cytokines downregulate the sarcoendoplasmic reticulum pump Ca2+ ATPase $2 \mathrm{~b}$ and deplete endoplasmic reticulum $\mathrm{Ca} 2+$, leading to induction of endoplasmic reticulumstress in pancreatic beta-cells. Diabetes 2005;54: 452-461.

33. Rowland AA, Voeltz GK. Endoplasmic reticulum-mitochondria contacts: function of the junction. Nat Rev Mol Cell Biol 2012;13:607-625.

34. Giorgi C, De Stefani D, Bononi A, et al. Structural and functional link between the mitochondrial network and the endoplasmic reticulum. Int J Biochem Cell Biol 2009;41:1817-1827.

35. Bravo R, Vicencio JM, Parra V, et al. Increased ER-mitochondrial coupling promotes mitochondrial respiration and bioenergetics during early phases of ER stress. J Cell Sci 2011;124:2143-2152.

36. Lynes EM, Raturi A, Shenkman $M$, et al. Palmitoylation is the switch that assigns calnexin to quality control or ER Ca2+ signaling. J Cell Sci 2013;126:3893-3903.

37. Fu S, Yang L, Li P, et al. Aberrant lipid metabolism disrupts calcium homeostasis causing liver endoplasmic reticulum stress in obesity. Nature 2011;473:528-531.

38. Arruda AP, Pers BM, Parlakgül G, et al. Chronic enrichment of hepatic endoplasmic reticulum-mitochondria contact leads to mitochondrial dysfunction in obesity. Nat Med 2014;20:1427-1435. 